\title{
Innovation des pratiques, Maître Formateur et accompagnement par la recherche
}

Jean-Michel Perez and Géraldine Suau

\section{(2) OpenEdition}

1 Journals

Electronic version

URL: http://journals.openedition.org/ripes/2171

DOI: $10.4000 /$ ripes. 2171

ISSN: 2076-8427

Publisher

Association internationale de pédagogie universitaire

Electronic reference

Jean-Michel Perez and Géraldine Suau, « Innovation des pratiques, Maître Formateur et accompagnement par la recherche », Revue internationale de pédagogie de l'enseignement supérieur [Online], 35(2) | 2019, Online since 13 March 2020, connection on 08 September 2020. URL : http:// journals.openedition.org/ripes/2171 ; DOI : https://doi.org/10.4000/ripes.2171

This text was automatically generated on 8 September 2020

Article L.111-1 du Code de la propriété intellectuelle. 


\title{
Innovation des pratiques, Maître Formateur et accompagnement par la recherche
}

\author{
Jean-Michel Perez and Géraldine Suau
}

\section{Introduction}

1 La question de l'inclusion est à considérer comme une des problématiques éducatives contemporaines dont l'enjeu nécessite un investissement de tous pour chacun, afin de permettre un accès équitable à l'éducation et à la formation (Akkari et Barry, 2018). La France, depuis 2005, n'échappe pas à cette mutation inclusive (Ployé, 2018) et met les enseignants comme les formateurs d'enseignants dans un contexte auquel ils n'ont pas été préparés.

2 En effet, enseigner, auprès d'un élève reconnu institutionnellement handicapé (ERIH) ne va pas de soi. D'abord parce que les professeurs d'école en France, n'ont pas une culture de l'inclusion, de par la construction même de leur métier, qui s'est produite en dehors des questions liées au handicap (Chauvière, 2012; Chauvière et Fablet, 2001; Chauvière et Plaisance, 2008). Ensuite, parce que les résistances mises en place comme «l'absence de formation », « la peur de ne pas savoir faire » (Mazereau, 2008, 2014), et/ ou les stratégies défensives qui « consistent à expulser du champ pédagogique l'élève handicapé perçu comme perturbateur ou encore à l'invibiliser » (Ployé, 2018, p. 142) sont nombreuses. Ensuite, parce que le changement attendu s'inscrit dans différentes dimensions du métier (pédagogique, didactique, coopérative, éthique...) et qu'il vient "déstabiliser les représentations et les hiérarchies instituées" (Mazereau, 2014, p. 26). Cette question est d'autant plus sensible pour les maitres formateurs. En effet, en France, les formateurs du premier degré ${ }^{1}$ que l'on nomme des Professeurs des Écoles Maîtres Formateurs (PEMF) accomplissent une mission d'enseignement et d'éducation auprès d'élèves, en tant qu'enseignant et contribuent en tant que formateur à la formation initiale et continue des enseignants et des futurs enseignants au sein d'un 
établissement d'enseignement supérieur qui est l'Institut National Supérieur du Professorat et de l'Éducation (INSPÈ). La circulaire du 18 octobre 2016, précise que ce sont « ces deux responsabilités qui font d'eux des acteurs essentiels de la formation des personnels enseignants et d'éducation : en tant qu'experts de la pratique de la classe, de la mise en œuvre de la politique éducative d'établissement et de suivi des élèves, de la polyvalence de leur métier, ils sont les garants d'une articulation (...) entre savoirs théoriques et pratique professionnelle et des relais pour faire connaître et diffuser les outils et les ressources institutionnelles». Les deux missions (d'enseignant et de formateur) sont donc articulées entre elles, mais elles s'appuient sur deux référentiels distincts : le référentiel de compétences professionnelles des métiers du professorat et de l'éducation ${ }^{2}$ qui constitue pour une part le cadre de référence pour orienter l'action enseignante, et le référentiel de compétences professionnelles du formateur des personnels enseignants et éducatifs ${ }^{3}$ pour l'accompagnement des élèves fonctionnaires stagiaires et des enseignants titulaires en reprise d'études. Comment dès lors, accompagner des élèves fonctionnaires stagiaires ou des enseignants notamment sur la compétence commune: "Prendre en compte la diversité des élèves ${ }^{4}$ alors que le "donner une place à l'élève ", et de surcroit celui de donner une place à un élève reconnu institutionnellement handicapé (Suau, 2016, 2018), constitue dans sa propre pratique de classe un problème professionnel?

4 Notre réflexion propose ainsi d'interroger, dans le cadre de l'enseignement supérieur, le développement praxéologique des formateurs d'enseignants qui reste peu étudié (Brau-Antony, 2013), notamment lors de réformes (Maylerot, 2012; Perez-Roux, 2012; Perez-Roux et Maleyrot, 2015) dans une démarche de recherches collectives, d'une «pratique du travail en groupe » (Ferry, 1971; Perez, 2018) entre chercheurs et PEMF, afin de répondre à une «logique de l'injonction et de l'exhortation» (Perez, 2015; Husson et Perez, 2016). Notre question de recherche est alors la suivante : en quoi un dispositif de recherche collectif peut-il favoriser le développement praxéologique des PEMF sur les enjeux posés par l'éducation inclusive?

5 Après avoir présenté les éléments théoriques et méthodologiques de notre étude, nous interrogeons à partir d'une étude de cas (Passeron et Revel, 2005) et dans le dispositif de recherche phénoméno-praxéologique (Assude, Perez, Suau et Tambone, 2018) le développement praxéologique d'un PEMF en lien avec le Domaine de Compétences 4 (DC4) : «Observer-Analyser-Évaluer » et spécifiquement «Observer et analyser des éléments de pratique professionnelle pour conseiller et aider à réajuster les pratiques ».

\section{La théorie anthropologique du didactique pour penser un dispositif de recherche et de développement professionnel}

6 Afin de décrire et d'analyser les pratiques professionnelles, tout en incluant les discours qui accompagnent les pratiques d'une PEMF, nous convoquons la notion de praxéologie de la théorie anthropologique du didactique (TAD). Dans cette théorie, toute activité humaine peut s'analyser en une succession de tâches, de divers types et à partir de trois autres composantes (Chevallard, 1998). Tout d'abord, la réalisation d'un type de tâche $\mathrm{T}$ suppose une technique $\tau$, c'est-à-dire une manière de faire pour réaliser ces tâches; ensuite, une technique est justifiée et rendue intelligible par une 
technologie $\theta$, soit par un discours technologique; enfin, la technologie est, elle même éclairée et justifiée par une théorie $\Theta$. Ainsi, autour d'un type de tâche, on trouve un triplet formé d'une technique, d'une technologie et d'une théorie. Le tout, constituant

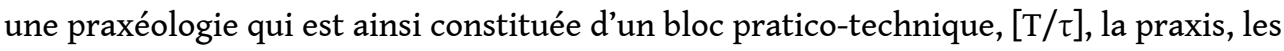
savoir-faire et d'un bloc technologico-théorique, $[\theta / \Theta]$, le logos, les savoirs.

7 Nous précisons également, que c'est par les institutions ${ }^{5}$ que les praxéologies parviennent jusqu'aux personnes: "On ne peut comprendre les apprentissages personnels si l'on ne cherche pas à comprendre les apprentissages institutionnels (...). Il y a, dans la diffusion des connaissances (et des ignorances) et des pratiques (et des incapacités), une dialectique indépassable entre personnes et institutions » (Chevallard, 2003).

8 Par ailleurs, et c'est un élément important de notre cadre théorique pour analyser l'évolution professionnelle corrélée à l'identification de pratiques innovantes, nous continuons à nous adosser à la TAD et à la théorie anthropologique de Mary Douglas (1999) qui interrogent la notion d'assujettissement aux institutions. En effet, pour ces deux théories, « Dès sa naissance, tout individu est assujetti à de multiples institutions, c'est-à-dire à la fois soumis à, et soutenu par des institutions, telle sa famille, dont il devient par là le sujet... D'une manière générale, c'est par ses assujettissements, par le fait qu'il est le sujet d'une multitude d'institutions, que l'individu $\mathrm{x}$ se constitue en une personne » (Chevallard, 1998, p. 2). C'est pour cela que nous avons produit un dispositif de recherche (pour plus de détail, se référer à Assude et al., 2018; Perez, Assude, Suau et Tambone, 2017; Suau, 2016) qui comporte plusieurs institutions: une institution d'action en classe, une institution d'analyse des praxéologies professionnelles, une institution de production de praxéologies professionnelles dans le groupe de recherche et une institution de transposition en formation. Ce dispositif constitue un milieu pour apprendre, notamment par un questionnement "en résonnance » engendré par les différents topos occupés dans ces institutions.

Nous situons pour cette production, notre observation dans l'institution d'analyse des praxéologies professionnelles, où le PEMF observe et analyse sa pratique et celle d'un pair. L'observation est instrumentée en référence aux entretiens en auto-confrontation (Clot, Faitä, Fernandez et Scheller, 2000; Clot et Leplat, 2005; Dubosq et Clot, 2010). Il s'agit d'abord d'enregistrer « les commentaires que le sujet, confronté aux images de sa propre activité, adresse au chercheur » (Clot et al., 2000, p. 4). C'est la confrontation à la pratique qu'il nous intéresse d'observer. Nous ne sommes pas dans une situation dégradée, ni dans une commande, et ce n'est pas le chercheur qui sélectionne un extrait, mais la PEMF qui choisit un extrait qu'elle trouve "remarquable», en ce sens qu'elle le remarque dans sa pratique, ce qui sous-entend qu'il y a un fort engagement, puisqu'elle a visionné la totalité de la séance et choisit un extrait. C'est pour cette raison que le chercheur lui demande en début d'analyse, d'expliciter le choix de l'extrait avec une seule question: «Pourquoi cet extrait-là?». Autrement dit, le chercheur ne l'invite pas «à décrire le plus précisément possible les gestes et les opérations observables sur l'enregistrement vidéo jusqu'à ce que les limites de cette description se manifestent, jusqu'à ce que la vérité établie soit prise en défaut dans la véracité du dialogue, par l'authenticité dialogique " (Dubosq et Clot, 2010, p. 265). Le chercheur dans notre dispositif est dans une position non directive, pour permettre à la PEMF de choisir ce qu'elle a à dire, parmi la multitude d'informations qu'elle a eu à disposition, hors de toute influence directe (les captations sont fournies à chaque 
PEMF). D'un point de vue matériel, ce n'est pas non plus le chercheur qui assure le défilement des images, les retours en arrière ou les arrêts de l'image et qui ponctue le discours du « sujet » (Clot et al., 2000, p. 5) mais la PEMF. Dans la relation dissymétrique chercheur-enseignant, c'est un moyen pour permettre à la PEMF de tenir le fil directeur de son discours, puisque que c'est elle qui se raconte en racontant son objet et c'est elle qui communique en raison ce qu'elle a pu comprendre de la situation éprouvée et observée.

Pour l'analyse croisée, il s'agit de reproduire ce qui est fait dans l'analyse simple, à partir d'un autre point de vue, celui d'un «pair PEMF ». Ce dernier regarde, dans un temps qui lui convient, la séance de son collègue et choisit un très court extrait qui répond à la consigne du "pour quoi faire?». C'est l'analyse faite sur l'extrait qui est portée à la connaissance du chercheur et du PEMF dans le cadre de l'analyse croisée. Le chercheur ne sollicite à aucun moment les commentaires des deux PEMF, mais s'intéresse à accueillir et à comprendre leurs discours, proche d'une écoute inconditionnelle de l'autre (Perez, 2018) en référence aux travaux de Roger (1942) et ce pour faire dynamique de changement. Nous pouvons aussi nous référer à Yvon et Garon (2006) qui témoignent de perspectives professionnelles de ce type d'analyse. Ces auteurs insistent en effet, sur le développement du commentaire chez les praticiens et leur contribution directe à développer et soutenir l'analyse de leur propre activité : "les conséquences de cette confrontation aux images de son activité sont incontestables : ouverture d'une zone de développement potentiel, développement de la pensée et du pouvoir d'agir, prise de conscience, interrogation, mention d'autres possibilités d'action et dialogue sur ce thème avec une collègue » (Yvon et Garon, 2006, p. 69).

11 Ce sont les points de vue différents racontés sur une même situation et accueillis de «manière inconditionnelle» par le groupe de recherche, qui sont ici les fondements propédeutiques de la co-construction d'une culture commune d'une "rechercheformation » (Perez, 2018; Macaire, 2018, 2019). Dans ce dispositif, il s'agit aussi de ne pas mésestimer l'influence majeure des institutions sur les sujets, puisque tout sujet - le chercheur tout comme l'enseignant - est agi par les institutions, mais peut aussi agir sur les institutions, ce qui amène dès lors à s'attacher aux rétroactions dans celles-ci pour faire « innovation ».

\section{Le recueil et le traitement des données}

12 Notre dispositif méthodologique est constitué de deux types de recueil de données, un en classe dans l'institution d'action classe et un après l'action, dans l'institution d'analyse des praxéologies professionnelles. Dans l'institution classe, nous faisons un enregistrement audio des entretiens ante $e^{6}$ et post séance et un enregistrement audiovidéo d'une séance d'enseignement et d'apprentissage, avec un plan large sur l'intégralité des élèves, ce qui nous permet de repérer les actions du PE dans la classe, un plan sur le PE pour pouvoir exploiter ses interactions avec les élèves, un plan sur l'élève reconnu institutionnellement handicapé et sur sa production et un plan sur l'environnement proche de l'élève reconnu institutionnellement handicapé, ce qui nous permet d'observer les interactions entre les membres du groupe et la production demandée. Dans l'institution d'analyse des praxéologies professionnelles, pour l'analyse simple, nous faisons deux enregistrements audio-vidéo, le premier en 
adoptant lors de cet enregistrement une position hors du cadre de l'image filmée, dans l'axe de l'objectif mais en contrechamp, afin de filmer le PEMF de face (Clot et al., 2000, p.5) et le second sur l'extrait choisi par le PEMF, qu'il est en train d'expliciter. Le chercheur est toujours hors du cadre. Pour l'analyse croisée, nous faisons également deux enregistrements audio-vidéo. Le premier en contrechamp en filmant les deux PEMF de face et en cadrant de façon serré, pour pouvoir analyser les éléments de proxémie et le second sur l'extrait commenté par le pair.

Pour traiter les différentes traces récoltées, nous procédons en deux temps. Un premier temps où afin de caractériser les savoir-faire et les savoirs professionnels nous articulons l'analyse des techniques mises en œuvre relatives au type de tâche T " donner une place à l'élève handicapé » dans l'institution d'action (I1) avec l'analyse les discours sur les pratiques dans l'institution d'observation et d'analyse des praxéologies professionnelles (I2). Pour ce faire, nous faisons le choix de nous situer dans une étude par cas individuels, caractéristique d'une approche clinique en sciences sociales, qui donne une primauté « de l'information et de l'observation portant sur la totalité des manifestations d'un être humain (...) placé en situation et en évolution » (Grawitz, 2015, p. 359). Ainsi, à partir des transcriptions ${ }^{7}$ de séances de classes, des entretiens ante et post, des productions des élèves et des fiches de préparation, nous faisons une présentation du contexte général, avec une description de la situation concernée, de la classe et des élèves observés, ainsi que le synopsis de la séance. Par synopsis, nous entendons dégager les phases de la séance (définition du jeu, travail en autonomie, mise en commun...), la temporalité de la séance et les modes de regroupement (collectif, individuel, petit groupe). Nous prenons dans notre recherche le synopsis comme une première structuration de la pratique qui "permet de commencer à saisir les grandes lignes du jeu didactique" (Sensevy, 2011, p.258). Ensuite, pour identifier les techniques de l'enseignant pour « donner une place à l'élève handicapé ", nous réduisons l'ensemble des observations dans la classe au récit d'un ensemble d'épisodes, qui constitue une intrigue, que nous pouvons définir en référence à Sensevy (2011) comme « un outil clinique, par sa manière d'approcher «les choses mêmes » et leur mouvement dans une forme (relative) de suspension du jugement et de neutralité. Elle peut être plus ou moins chargée en termes théoriques, mais dans chaque cas, elle propose une détermination relative à quoi jouent les transactants... » (Sensevy, 2011, p. 270). Nous passons ensuite pour chaque étude de cas à l'analyse des discours sur les pratiques dans l'institution d'observation et d'analyse des praxéologies professionnelles (I2). Pour cela nous nous appuyons sur les transcriptions des analyses simple et croisée. Et, nous précisons les discours des techniques et les discours sur les techniques relatives au type de tâche «donner une place à l'élève » qui justifient, produisent, rendent intelligible les techniques mises en œuvre, au sens de technologie de Chevallard (1998).

14 À partir de ces premiers éléments, nous identifions le développement praxéologique et les effets de notre dispositif bi-dimensionnel. Nous considérons compte tenu de notre question de recherche, qu'il est important de tenir compte de deux propriétés, celle de la temporalité et celle de la singularité (Pastré, 2011). Ainsi, concernant l'analyse de l'évolution des praxéologies professionnelles, nous nous appuyons sur les différentes situations et les transcriptions des analyses simples et croisées, dans une dimension temporelle. Et nous repérons des épisodes qui traduisent « le cheminement » des PEMF, ce dernier caractérisê par l'évolution à la fois de leur praxis et de leur logos 
" relativement à l'observation et à l'analyse des éléments de pratique professionnelle » et en lien avec le type de tâche "donner une place à l'ERIH ». Ces épisodes sont consignés dans un ordre chronologique.

Les données sur lesquelles nous nous appuyons pour cette production sont les analyses simples (AS) et croisées (AC) sur 6 séances de la PEMF H. qui ont fait l'objet d'un recueil sur trois années, du 7 octobre 2013 au 1er juillet 2016.

La PEMF dont nous observons les praxéologies est professeure des écoles depuis 1998. Elle a enseigné pendant douze ans avant de devenir directrice d'école, puis professeure des écoles maître-formateur. En 2013, elle intègre une école d'application ${ }^{8}$. Concernant son implication dans ce projet, elle l'explicite au regard de l'accueil des Élèves Reconnus Institutionnellement Handicapé (ERIH) en milieu ordinaire et pour lesquels « elle ne sait pas trop bien ce qu'elle peut faire». Nous montrerons que ses techniques d'observation et d'analyse de sa pratique et de celle d'un tiers, dans le cadre de l'accueil de la diversité des élèves ne vont pas de soi, ce qui peut alors venir questionner ses praxéologies professionnelles dans sa double mission.

\section{4. Étude des techniques d'observation et d'analyse des éléments de pratique professionnelle chez un maitre formateur pour « prendre en compte la diversité des élèves »}

17 Dans ses premières analyses, la PEMF $\mathrm{H}$. considère l'ERIH comme un élève qui fait l'objet d'injustice (S1) et comme un élève imitateur qui « imite les adultes », « qui imite les gestes » dans le cadre de son analyse simple (S2, AS, Tdp.12). Dans l'analyse croisée, avec la PEMF Pair sur la situation «Technique opératoire de l'addition posée » (S2), la PEMF H. réaffirme son analyse en lien avec les gestes d'imitation mais la PEMF Pair a observé contrairement à la PEMF H. que cet élève était dans la tâche tout au long de la situation. Un échange s'engage alors entre les deux, dans lequel la PEMF Pair montre les différents empêchements produits qu'elle a pu repérer mais aussi la méthode que l'élève a choisie pour dénombrer. Confrontée à un regard différent (appuyé par le support de la vidéo) cela vient bousculer l'analyse de la PEMF H. qui modifie progressivement celle-ci. C'est ce qui l'amène à énoncer que c'est « hyper intéressant par rapport à sa pratique» (AC, Tdp.16) et que c'est bien le visionnage de la séance qui lui permet non seulement de repérer l'élève dans la tâche mais également que son observation n'était pas complète : "C'est en décortiquant la séance que tu te rends compte de tout ce à coté de quoi tu passes » (AC, Tdp.16).

18 En revanche, dans le cadre de l'analyse simple de S3 «Robots », la PEMF H. décide de présenter deux extraits où l'ERIH a pris position dans sa place d'élève. Elle le présente comme un élève qui s'inscrit d'abord dans la situation en individuel (AS, Tdp.1) et ensuite en collectif, en précisant «qu'il intervient à bon escient ». Son discours dans cette analyse montre qu'elle porte un regard sur l'élève Mathias, comme un élève qui peut prendre position. Elle choisit en effet de ne pas montrer les moments où l'élève a des difficultés pour assumer son topos d'élève et elle affirme (AS, Tdp.5) : "J'imagine qu'il avait bien compris la situation. Car maintenant, je sais qu'en fait, ça ne lui pose pas de souci de résoudre une énigme de mathématiques et qu'il est à l'aise par rapport à cela ». Ce qu'elle vient confirmer dans son discours dans S5 où elle présente l'ERIH dans l'analyse simple et 
croisée, comme un élève qui est en capacité de résoudre une situation mathématique (AS, Tdp.6) : "Moi je me suis ciblée sur le travail de groupe, Mathias n'est pas forcement en difficulté, il aurait été capable de le faire, d'investir la situation et là il s'est cantonné à un rôle de colleur'. Et c'est long comme séance et il y a beaucoup de temps euh, c'est long; on a envie de dire pourquoi autant de temps pour ça; on aurait pu le faire plus rapidement sans tout ce protocole de groupe ». C'est ce qui l'amène dans son échange avec la PEMF pair à préciser, contrairement à ce que cette dernière énonce, que le peu de participation de Mathias est plus en lien avec la situation, le contrat proposé et le respect de celui-ci par l'élève. Pour la PEMF H., c'est en effet, le fait que l'élève n'ait qu'un rôle de colleur qui l'amène à ne pas s'investir dans la situation et à attendre de pouvoir coller. Il a compris selon la PEMF H. les enjeux, ce qu'elle vient argumenter avec le fait que dès qu'elle est présente dans le groupe il arrête de jouer pour être attentif à ce qu'elle dit (AC, Tdp.19) : «Pour moi, ce que j'avais dit, c'est qu'il y avait une histoire de contrat de travail au départ : il a investi son rôle. Il savait qu'il était colleur, il a pris sa colle. Et après il s'en est plus, il n'a plus démordu de ce rôle qu'il allait avoir. Il allait coller. À la fin, il allait coller...Et Mathias, lui, il alterne les phases de "je joue " ... Et quand la maitresse arrive, je "pense effectivement " ... et puis dès que j'ai le dos tourné, hop. C'est parti. Il avait très bien investi son rôle d'élève effectivement ».

19 La PEMF H. porte également un regard sur les situations mathématiques proposées à l'ERIH et les interroger au regard de ses choix. Elle présente d'abord un extrait en lien avec le choix des nombres où elle pointe que les nombres proposés pour les additions en colonne $15+21$ et $32+23$ peuvent se lire horizontalement et verticalement. C'est ce qui amène la PEMF $\mathrm{H}$. dans l'analyse simple à énoncer au chercheur que : "Là il y a une erreur de ma part sur le choix des nombres » et de rajouter "ça c'est clair... Que je les lise dans n'importe quel sens " (AS, Tdp.22). Dans cette même séance, elle s'interroge également sur la nécessité de faire manipuler les élèves car elle s'aperçoit que cela n'a rien changé. C'est ce qui l'amène à questionner non seulement l'adéquation entre ses objectifs dans cette situation et le choix de manipulation pour les élèves mais aussi sa conception même de la manipulation, qu'elle associe au fait que les élèves sont petits et qu'il est difficile pour eux de construire des apprentissages s'ils ne manipulent pas (AS, Tdp.26) : "J'avais choisi de donner du matériel à certains élèves pour les aider dans la construction de la technique de l'addition, en fait ça ne servait à rien du tout. L'objectif était d'arriver justement à poser une technique et ce n'était pas, il ne pouvait pas la découvrir. Puisque mon objectif c'était d'arriver à la technique pas la compréhension à proprement parler de l'échange d'unité en dizaine. Soit mon objectif était mal ciblé au vu de la manipulation que je leur faisais faire, soit l'inverse. Au final, je pense j'aurai un peu tendance à me dire il est petit, il est en difficulté, il faut qu'il manipule, c'était un peu voilà, je caricature ».

Dans le cadre de l'analyse croisée sur cette même séance, alors que la PEMF pair choisi d'échanger sur le rôle de l'aide humaine, la PEMF $H$. après avoir repéré et acquiescé à l'analyse de son pair, décide de questionner à nouveau, ce qu'elle a observé et qui semble l'avoir perturbé dans la séance, les nombres proposés aux élèves, notamment en disant: "Et en plus regarde cela. Regarde ces nombres-là: $32+23,15+21$, mais c'est hallucinant" (AC, Tdp.37). Elle lui détaille là ce qu'elle décrit comme une erreur, d'autant plus que certains élèves ne savent pas écrire les nombres en colonne, et presque sidérée, elle continue en prenant une feuille et en montrant à son pair. Dans le cadre de S5 et S6, la PEMF H. choisit dans l'analyse simple de questionner la situation au regard de la forme d'étude proposée : fallait-il proposer un travail de groupe pour cette situation mathématique? Elle pose là à haute voix ce qui l'interroge par rapport à sa pratique, mais ne propose pas pour autant de réponses. C'est dans le cadre des deux 
analyses croisées avec son pair qu'elle réinterroge cela et commence à réfléchir à de possibles réponses. D'abord, avec le choix d'introduire des modalités pédagogiques, comme des objets dans le milieu qui peuvent finalement faire obstacle en empêchant l'élève de jouer ( $55 / \mathrm{AC}, \mathrm{Tdp} .33$ ), ensuite, avec la construction même de la situation et sur ce qui a été demandé aux élèves. Elle choisit de l'expliciter par rapport au dénombrement des voitures dans le garage (premier rôle), qui est demandé en groupe mais qui après analyse lui parait difficile à réaliser (AC, Tdp.19) : «En fait, la première activité, c'était de compter les voitures et compter à quatre alors, je suis revenu sur le fond de ce qu'on avait imaginé de demander aux élèves quoi. Compter à quatre des voitures dans un garage, je ne vois pas comment ils pouvaient s'organiser. Il fallait du coup, qu'il y en ait qu'un qui le fasse quoi. Ou alors, se passer le travail en cours de route: attention, moi j'en ai compté 22, continue..., mais c'est, juste pas possible quoi... c'est une activité qui se fait toute seule quoi (...)». C'est sur cette argumentation - d'une situation mal construite avec une succession d'étapes pour accéder à la résolution, qu'elle redétaille à son pair - qu'elle s'appuie pour justifier que l'ERIH, joue avec les voitures et que cela a été compliqué pour lui, d'être dans l'activité, comme pour d'autres élèves de la classe.

21 Les discours de la PEMF H. ne témoignent pas dans un premier temps, de traces d'une observation concernant la prise en compte de la diversité. Elle centre certes son observation sur l'élève, mais pour le qualifier de perturbateur ou d'imitateur avec un discours référant à un registre qui relève plutôt de l'affect. Elle semble même avoir quelques difficultés pour repérer dans S2, que l'élève s'inscrit dans la situation et qu'il fait l'objet d'empêchements par un tiers. L'analyse proposée peut traduire une absence de techniques et de discours relativement à un type de tâche, ici une observation fine des pratiques. Cela vient donc questionner dans un premier temps la capacité de la PEMF à observer ses pratiques, les pratiques d'un tiers et donc sa mission même «d'observation et d'analyse des éléments de pratique professionnelle pour conseiller et aider à réajuster les pratiques ». Mais au fil des observations, des analyses et des échanges avec son pair, ses praxéologies évoluent et les élèves deviennent dans son discours capable de résoudre une situation mathématique. L'analyse des éléments de pratiques professionnelles s'affine notamment sur le choix de la situation d'enseignement proposée, le choix des nombres qui peuvent faire obstacle, la question de la manipulation qui nécessite d'être interrogée et le choix de la forme d'étude, tout comme sur les objets introduits dans le milieu.

\section{Conclusion}

Le choix d'utiliser le cadre de la théorie anthropologique du didactique (Chevallard, 1998) pour analyser le développement des praxéologies professionnelles d'une professeure des écoles maitre formateur, nous paraît intéressant d'abord dans la possibilité de décrire assez finement des savoir-faire et des savoirs professionnels, ensuite pour repérer spécifiquement les composantes qui font obstacles et cela, aussi bien dans les situations d'action que dans les situations après l'action (Suau, 2019).

24 Nous avons recentré notre recherche sur une praxis, au centre de l'accompagnement des enseignants stagiaires, qui consiste à être capable d'observer et d'analyser des éléments de pratique professionnelle. Pour rendre compte de ses praxéologies effectives, nous avons exploité les analyses simples et les analyses croisées dans notre dispositif, où les institutions ont une place prépondérante, ce qui nous a permis de montrer qu'il y avait peu de technologies, soit de discours sur les techniques 
(Chevallard, 1998) pour la PEMF concernée par l'étude et qu'il n'était pas aisé pour elle dans les premières séances d'avoir une observation fine de l'élève dans sa pratique et ni dans celle d'un tiers. Nous avons dans un second temps montré qu'une évolution des praxéologies était possible relativement à l'observation de la relation élève-enseignant et à l'analyse des éléments de pratiques professionnelles et qu'il était développé un outillage praxéologique nécessaire en tant «qu'expert de la pratique de classe », pour répondre à la diversité et aux besoins des apprenants.

L'accompagnement par la recherche pour les praticiens-formateurs nous semble ainsi être une occasion pour faire évoluer la praxis de ces acteurs. Ces derniers considérés comme essentiels dans la formation des personnels enseignants sont en effet soumis à de nombreux changements institutionnels qui conduisent à de nécessaires réorientations. Comme cela a pu être le cas avec la réforme de la masterisation où les enseignants devaient obtenir un master pour pouvoir enseigner, ce qui nécessitait alors de développer pour les formateurs de nouvelles praxéologies, en lien avec l'initiation à la recherche, la préprofessionnalisation avec les stages etc. (Perez-Roux et Maylerot, 2015) et comme cela semble être encore aujourd'hui le cas avec le contexte de l'école inclusive. Le problème étant pour eux de contribuer à l'insertion professionnelle des enseignants stagiaires ce qui suppose de mobiliser des ressources et des praxéologies adaptées, ce qui peut paraitre difficile si nous ne leur donnons pas les moyens pour résoudre les problèmes liés à des situations de travail inhabituelles (Brau-Antony et Mieusset, 2013).

Par contre, ce type d'accompagnement où l'activité réflexive et le jeu d'institutions sont au centre de l'évolution des praxéologies ne peut que profondément interroger l'épistémologie de la recherche et donc le positionnement et les attitudes des chercheurs dans la pratique du travail en groupe au service du devenir de l'élève. Cette hypothèse d'une influence du chercheur lui-même - par sa responsabilité de construction d'un milieu où chacun peut assumer son topos en fonction des institutions dans lesquelles il est, et devenir producteur de praxéologies nouvelles - sur la connaissance et les usages à propos de l'éducation et des pratiques Inclusives... (Perez, 2018), nous incite à continuer nos travaux en ce sens, pour accompagner le changement collectif et pour pouvoir répondre à la diversité des élèves.

\section{BIBLIOGRAPHY}

Akkari, A. et Barry, V. (2018). Pour une école inclusive : des intentions aux réalisations. Revue internationale d'éducation de Sèvres, 78, 37-46.

Assude, T., Perez, J.-M., Suau, G. et Tambone, J. (2018). Effets d'un dispositif de recherche sur les pratiques inclusives en milieu scolaire ordinaire. Revue des Sciences de l'Éducation, 44(1), 105-137.

Brau-Antony, S. (2013). La place de la recherche dans le développement professionnel des formateurs IUFM. Tréma, 39, 23-34. 
Brau-Antony. S. et Mieusset C. (2013) Accompagner les enseignants stagiaires : une activité sans véritables repères professionnels. Recherche et formation, 72, 27-40.

Chauvière, M. (2012). D'un contentieux historique à une culture partagée. La nouvelle revue de l'adaptation et de la scolarisation, 57, 45-54.

Chauvière, M. et Fablet, D. (2001). L'instituteur et l'éducateur spécialisés. D'une différenciation historique à une coopération difficile. Revue française de pédagogie, 134, 71-85.

Chauvière, M. et Plaisance, E. (2008). Les conditions d'une culture partagée. Reliance, 27, 31-44.

Chevallard, Y. (1998). Dictionnaire de didactique des mathématiques, 1997-1998. Organisations

didactiques : 1 . les cadres généraux. Repéré à http://yves.chevallard.free.fr/spip/spip/IMG/pdf/

Organisations_didactiques_1_1998_.pdf

Chevallard, Y. (2003, 20 et 21 mars). Vingt ans de recherche en didactique de l'Éducation Physique et Sportive à l'INRP (1983-2003). Bilan et perspectives. Journées d'études INRP-GÉDIAPS. Didactique et formation des enseignants, Paris, France.

Clot, Y., Faita, D., Fernandez, G. et Scheller, L. (2000). Entretiens en autoconfrontation croisée : une méthode en clinique de l'activité. Perspectives interdisciplinaires sur le travail et la santé, 2(1). repéré à http://pistes.revues.org/3833

Clot, Y. et Leplat, J., (2005). La méthode clinique en ergonomie et en psychologie du travail. Le travail humain, 68, 289-316.

Dubosq, J. et Clot, Y. (2010). L'autoconfrontation croisée comme instrument d'action au travers du dialogue : objets, adresses et gestes renouvelés. Revue d'anthropologie des connaissances, 4(2), 255-286.

Douglas, M. (1999). Comment pensent les institutions. Paris, France : La découverte.

Ferry, G. (1971). La pratique du travail en groupe. Paris, France : Dunod.

Grawitz, M. (2015). Méthodes des sciences sociales. (11 éd.). Paris, France : Éditions Dalloz.

Husson, L. et Perez, J.-M. (2016). Handicap et inclusion à l'école : entre mondialisation des droits et agir éducatif. Carrefours de l'Éducation, 42, 185-198.

Loi $n^{\circ}$ 2013-595 du 8 juillet 2013 d'orientation et de programmation pour la refondation de l'école de la République. Paris, France : Journal officiel.

Macaire, D. (2018). Accompagnement d'une équipe éducative par des chercheurs - Coopération et recherche collaborative entre je et nous. Dans P.-J. Laffitte (dir.), Coopération, éducation, formation (p. 171-179). Paris, France : L'Harmattan.

Macaire, D. (2019). La recherche-formation convient-elle pour orienter les pratiques dans le sens de l'accessibilité scolaire de jeunes élèves à besoins particuliers? La nouvelle revue - Éducation et société inclusives, 86(2), 79-92. doi:10.3917/nresi.086.0079

Maleyrot, E. (2012). Ruptures et transformations identitaires des maîtres formateurs face aux réformes de la formation des enseignants. Les Sciences de l'Éducation pour l'Ère Nouvelle, 45(3), 65-88.

Mazereau, P. (2014). Inclusion scolaire et nouvel ordre des savoirs : vers des professionnalités enrichies. La Nouvelle Revue de l'Adaptation et de la Scolarisation, 65, 21-30.

Mazereau, P. (2008). De l'intégration à la scolarisation des élèves handicapés : état des lieux et nouveaux besoins de formation des enseignants. Caen, France : CERSE.

Passeron, J.-C. et Revel, J. (2005). Penser par cas. Paris, France : Éditions de l'EHESS. 
Pastré, P. (2011). La didactique professionnelle. Approche anthropologique du développement des adultes. Paris, France : PUF.

Pelgrims, G. et Bauquis, C., (2016). Des « élèves à BEP » à la notion de besoins pédagogiques et didactiques particuliers pour apprendre des savoirs en classe ordinaire. Dans G. Pelgrims et J.-M. Perez (dir.), L'école dite inclusive? Injonctions, conceptions et pratiques effectives (p. 73-96). Suresnes, France : INSHEA.

Perez, J.-M. (2015). Normes et usages dans l'institution éducative : sur la notion d'inclusion scolaire. Dans Go, H. L. (dir.), Normes pour apprendre (p. 69-87). Nancy, France : PUN-Édulor.

Perez, J.-M., (2018). Éducation et pratiques inclusives : la pratique du travail en groupe et le « chercheur apprenant ». Note de Synthèse d'Habilitation, Université de Lorraine. École doctorale. ED 78. Sociétés, Langages, Temps, Connaissances (SLTC). Nancy.

Perez, J.-M., Assude, T., Suau, G. et Tambone, J. (2017). Usages de la vidéo dans un dispositif de recherche phénoméno-praxéologique : quelques effets sur les pratiques enseignantes inclusives. La nouvelle revue de l'adaptation et de la scolarisation, 78, 171-184.

Perez-Roux, T. (2012). Des formateurs d'enseignants à l'épreuve d'une réforme : crise(s) et reconfigurations potentielles. Les Sciences de l'Éducation pour l'Ère Nouvelle, 45(3), 39-63.

Perez-Roux T. et Maleyrot, E. (2015). L'accompagnement des enseignants à l'heure des réformes : stratégies des formateurs de terrain face à de nouvelles formes de (dé)professionnalisation. Éducation et socialisation, 38, 2-12.

Ployé, A. (2018). L'inclusion scolaire en France, un processus inachevé. Revue internationale d'éducation de Sèvres, 78, 137-146.

Rogers, C. (1942). Counseling and psychotherapy. Boston, MA : Mifflin.

Sensevy, G. (2011). Le sens du savoir. Bruxelles, Belgique : De Boeck.

Suau, G. (2016). Pratiques inclusives en mathématiques d'enseignants de classe ordinaire dans le premier degré (thèse de doctorat inédite). Université de Lorraine, Nancy, France.

Suau, G. (2018). Donner une place à l'élève reconnu handicapé en contexte scolaire ordinaire, quelles praxéologies professionnelles? La Nouvelle Revue Éducation et Société Inclusives, 81, 267-281.

Suau, G. (2019). La notion d'organisation praxéologique de la TAD pour analyser le développement professionnel. La Nouvelle Revue Éducation et Société Inclusives, 86, 93-106.

Yvon, F. et Garon, R. (2006). Une forme d'analyse du travail pour développer et connaître le travail enseignant : l'autoconfrontation croisée. Recherches qualitatives, 26, 51-80.

\section{NOTES}

1. Ces formateurs dit de "terrain" interviennent avec les formateurs conseiller pédagogique de circonscription et les formateurs INSPE auprès des futurs enseignants sur un tiers temps de leur service, ils sont titulaires d'un certificat d'aptitude aux fonctions d'instituteur ou de professeur des écoles maître formateur.

2. Fixé par l'arrêté du 1er juillet 2013 et la note de service n² 2015-055 du 17 mars 2015.

3. Les formateurs doivent maîtriser les compétences professionnelles décrites dans le référentiel annexé aux circulaires $n^{\circ} 2015-109$ du 21 juillet 2015 et $n^{\circ} 2015-110$ du 21 juillet 2015. Il est également attendu d'eux qu'ils poursuivent une démarche 
individuelle de formation dans les domaines qui relèvent de leur(s) champ(s) d'intervention.

4. Bulletin officiel no 30 du 25 juillet 2013 https://www.legifrance.gouv.fr/affichTexte.do? cidTexte=JORFTEXT000027721614\&dateTexte $=\&$ categorieLien=id

5. Concernant la notion d'institution, Chevallard se réfère à l'utilisation qu'en fait l'anthropologue Mary Douglas (1999). Ainsi « une institution I est un dispositif social total, qui peut certes n'avoir qu'une extension très réduite dans l'espace social (il existe des " microinstitutions »), mais qui permet - et impose - à ses sujets, c'est-à-dire aux personnes x qui viennent y occuper les différentes positions $\mathrm{p}$ offertes dans I ... Ainsi la classe est-elle une institution, de même que l'établissement ... » (Chevallard, 2009, p. 2).

6. Nous demandons aussi aux enseignants leur fiche de préparation, ce qui nous permet de repérer les objectifs de la séance, le matériel utilisé et le type de consigne qui est communiqué aux élèves.

7. Nous transcrivons l'intégralité des interventions orales (nous ne modifions pas les termes). Nous ne signifions pas les intonations, les accentuations, les pauses, ni les rires... Nous marquons, par contre, les moments inaudibles en écrivant inaudible dans la transcription. Nous faisons également apparaître le déroulement temporel de la séance ou des analyses simples et croisées en minutes et les tours de paroles.

8. Les écoles d'application étaient historiquement des annexes des écoles normales, où l'on formait les enseignants. Aujourd'hui, une école d'application accueille des enseignants en formation mais aussi des étudiants de l'ESPE et des professeurs étrangers. Elles ont comme titulaire des maîtres-formateurs, le directeur de l'école étant également un maître-formateur.

9. Pour l'enseignantes, Mathias n'investit pas la situation, puisqu'il n'assume pas de rôles épistémiques, mais qu'il choisit d'assumer un rôle de colleur qui est un rôle plus matériel

\section{ABSTRACTS}

en

Our article deals with teachers in Master Teacher Training Schools (MTTS), considered essential in the French education system for their teaching and training mission and subject to an institutional context that gives a significant weight to their commitment to pedagogical innovation. We propose to use a case study to examine the praxeological development of a teacher trainer who has been little studied in the context of a research training program based on the framework of the anthropological theory of didactics (Chevallard, 1998).

fr

Notre article concerne des Professeurs des Écoles Maîtres Formateurs (PEMF), considérés comme essentiels dans le système éducatif français pour leur mission d'enseignement et leur mission de formation et soumis à un contexte institutionnel qui donne un poids important à leur engagement dans des démarches d'innovation pédagogique. Nous proposons d'interroger à partir d'une étude de cas le développement praxéologique d'un formateur d'enseignants qui reste peu étudié dans un Dispositif de Recherche Faisant Formation à partir du cadre de la théorie anthropologique du didactique (Chevallard, 1998). 
INDEX

Mots-clés: innovation pédagogique, professeur des écoles, maître formateur, dispositif de recherche faisant formation, accompagnement par la recherche

\section{AUTHORS}

\section{JEAN-MICHEL PEREZ}

EA 2310 LISEC, Université de Lorraine, jean-michel.perez@univ-lorraine.fr

GÉRALDINE SUAU

EA 2310 LISEC, Université de Lorraine, geraldine.suau@univ-lorraine.fr 